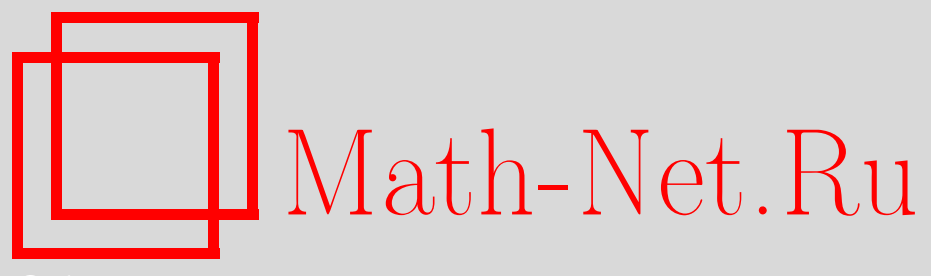

В. М. Кесельман, Об изопериметрическом неравенстве на конформно-параболических многообразиях, УМH, 2007, том 62, выпуск 6, 177-178

DOI: https://doi.org/10.4213/rm8540

Использование Общероссийского математического портала Math-Net.Ru подразумевает, что вы прочитали и согласны с пользовательским соглашением http://www . mathnet.ru/rus/agreement

Параметры загрузки:

IP: 54.162 .127 .20

26 апреля 2023 г., 17:51:01

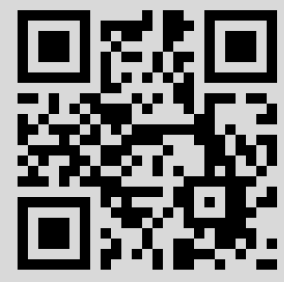




\section{Об изопериметрическом неравенстве на конформно-параболических многообразиях}

\section{В. М. Кесельман}

Прежде чем анонсировать основной результат, напомним некоторые понятия и договоримся об обозначениях.

Всюду ниже $\left(M^{n}, g\right)-n$-мерное некомпактное риманово многообразие без края. Для области $D \subset M^{n}$ через $V_{g}(D)$ и $S_{g}(\partial D)$ обозначим соответственно ее объем и площадь ее границы $\partial D$ в метрике $g$. Рассматриваемые области всегда будем предполагать предкомпактными и с гладкими границами.

Функция $P: \mathbb{R}_{+} \rightarrow \mathbb{R}_{+}$называется изопериметрической функцией многообразия $\left(M^{n}, g\right)$, если для любой области $D \subset M^{n}$ имеет место соотношение $P\left(V_{g}(D)\right) \leqslant$ $S_{g}(\partial D)$, называемое изопериметрическим неравенством.

Разумеется, интересна лишь нетривиальная, т. е. отличная от нуля, изопериметрическая функция, если таковая существует. В этом случае есть и нетривиальная максимальная изопериметрическая функиия (представляющая основной интерес)

$$
P_{\max }(x):=\inf _{V_{g}(D)=x}\left\{S_{g}(\partial D)\right\},
$$

называемая также изопериметрическим профилем многообразия.

Например, максимальная изопериметрическая функция (с точностью до постоянного положительного множителя $c$ ) для евклидова $n$-мерного пространства $\mathbb{R}^{n}$ имеет вид $P_{\max }(x)=c x^{(n-1) / n}$, а для пространства Лобачевского $\mathbb{H}^{n}-$ линейный вид $P_{\max }(x)=c x$.

Изопериметрическая функция многообразия, вообще говоря, зависит от его метрики.

Рассмотрим на многообразии $\left(M^{n}, g\right)$ класс метрик $\tilde{g}$, конформно эквивалентных его исходной метрике $g$, т. е. $\tilde{g}=\lambda^{2} g$, где $\lambda=\lambda(x)$ - положительная функция на многообразии $M^{n}$.

Нас интересует вопрос о том, к какому простейшему виду приводится максимальная изопериметрическая функция некомпактного риманова многообразия конформной заменой его исходной метрики.

Высказанная в [1] гипотеза состоит в следующем.

Некомпактные римановы многообразия разбиваются на два класса: конформнопараболические (если конформная емкость абсолюта многообразия равна нулю) и конформно-гиперболические (если указанная емкость положительна). Подробности см. в [1].

В первом классе лежит, например, пространство Евклида $\mathbb{R}^{n}$, во втором - пространство Лобачевского $\mathbb{H}^{n}$.

Утверждается, что для некомпактного многообразия найдется метрика $\tilde{g}$, конформно эквивалентная его исходной метрике, такая, что $\tilde{g}$-объем многообразия бесконечен, а изопериметрическая функция в метрике $\tilde{g}$ имеет тот же вид (с точностью до постоянного положительного множителя), какой она имеет в пространстве Евклида или в пространстве Лобачевского, в зависимости от конформного типа данного многообразия.

Для многообразий конформно-гиперболического типа гипотеза была сначала доказана в работе [1] при дополнительном условии отграниченности от нуля объема рассматриваемых областей. Затем в работе [2] от этого ограничения удалось освободиться.

Работа выполнена при поддержке РФФИ (грант № 05-01-01051). 
Следующая теорема дает подтверждение гипотезы для конформно-параболических многообразий, но пока при том же, что и выше, дополнительном условии. Возможно, что и в рассматриваемом случае оно окажется излишним.

Теорема. Предположим, что многообразие $\left(M^{n}, g\right)$ является конформно-параболическим. Фиксируем произвольно число $\varepsilon>0$. Тогда справедливы следующие утверждения.

i) Существует конформно эквивалентная исходной метрике $g$ полная риманова метрика $\tilde{g}$ на $M^{n}$, в которой обвем многообразия $M^{n}$ бесконечен и выполняется изопериметрическое неравенство евклидова вида:

$$
C V_{\tilde{g}}^{(n-1) / n}(D) \leqslant S_{\tilde{g}}(\partial D)
$$

для всех областей $D \subset M^{n}$, обвем $V_{\tilde{g}}(D)$ которых больше $\varepsilon$.

ii) При этом неравенство (1) является асимптотически точным в том смысле, что неравенство

$$
S_{\tilde{g}}(\partial D)<(1+\varepsilon) C V_{\tilde{g}}^{(n-1) / n}(D)
$$

c той же, что и в неравенстве (1), постоянной $C=C(\varepsilon)$ выполняется для всех областей $D$ некоторого исчерпания многообразия $M^{n}$.

Под исчерпанием многообразия $M^{n}$ здесь понимается непрерывное семейство $\{D(t)\}, t \in \mathbb{R}$, областей $D(t) \subset M^{n}$ таких, что $D\left(t_{1}\right) \Subset D\left(t_{2}\right)$ при любых $t_{1}<t_{2}$ и $\bigcup_{t} D(t)=M^{n}$.

В отношении максимальной изопериметрической функции многообразия теорему можно переформулировать следующим образом.

Если многообразие $\left(M^{n}, g\right)$ является конформно-параболическим, то для любого числа $\varepsilon>0$ в классе метрик на $M^{n}$, конформно эквивалентных метрике $g$, найдется полная метрика $\tilde{g}$, в которой $V_{\tilde{g}}\left(M^{n}\right)=+\infty$ и максимальная изопериметрическая функция $P_{\max }$ многообразия $M^{n}$ удовлетворяет неравенству

$$
C x^{(n-1) / n} \leqslant P_{\max }(x)<(1+\varepsilon) C x^{(n-1) / n}
$$

при всех значениях $x \in(\varepsilon,+\infty)$.

Доказательства приведенных результатов в идейном плане близки к изложенным в [1] и [2] в том смысле, что тоже опираются на конформную инвариантность конформной емкости. Однако нам долго не удавалось преодолеть специфические трудности, возникающие в случае многообразий конформно-параболического типа.

В заключение выражаю искреннюю благодарность В. А. Зоричу за научное общение, в результате которого и появилась эта работа.

\section{Список литературы}

[1] В. А. Зорич, В. М. Кесельман, Функи. анализ и его прил., 35:2 (2001), 12-23; англ. пер.: V. A. Zorich, V. M. Kesel'man, Functional Analysis and Its Applications, 35:2 (2001), 90-99. [2] В. М. Кесельман, Mam. сб., 194:4 (2003), 29-48; англ. пер.: V. M. Kesel'man, Sb. Math., 194:4 (2003), 495-513.

В. M. Кесельман (V. M. Keselman)

Московский государственный индустриальный университет

E-mail: kvm@glasnet.ru, kvlm@online.ru
Представлено А. Г. Сергеевым Принято редколлегией 09.10.2007 